This document is the unedited Author's version of a Submitted Work that was subsequently accepted for publication in ACS Applied Materials and Interfaces, copyright (c) American Chemical Society after peer review. To access the final edited and published work see:

https://dx.doi.org/10.1021/acsami. 7b15855. 


\title{
Photochemically activated motors: from electrokinetic to dif- fusion motion control
}

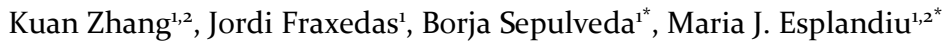 \\ ${ }^{1}$ Catalan Institute of Nanoscience and Nanotechnology (ICN2), CSIC and BIST, Campus UAB, Bellaterra, o8193 Barcelona, Spain \\ ${ }^{2}$ Universitat Autònoma de Barcelona (UAB), Bellaterra, E-o8193, Spain \\ KEYWORDS: Catalytic motors, Photoactivation, Chemomechanical actuation, Electro-hydrodynamics forces, Light- \\ driven motors
}

\begin{abstract}
Self-propelled micro/nano-motors that can transform chemical energy from the surrounding environment into mechanical motion are cutting edge nanotechnologies with potential applications in biomedicine and environmental remediation. These applications require full understanding of the propulsion mechanisms to improve the performance and controllability of the motors. In this work, we demonstrate that there are two competing chemomechanical mechanisms at semiconductor/metal $(\mathrm{Si} / \mathrm{Pt})$ micromotors in a pump configuration under visible light exposure. The first propulsion mechanism is driven by an electro-osmotic process stemmed from a photoactivation reaction mediated by $\mathrm{H}_{2} \mathrm{O}_{2}$, which takes place in two separated redox reactions at the $\mathrm{Si}$ and $\mathrm{Pt}$ interfaces. One reaction involves the oxidation of $\mathrm{H}_{2} \mathrm{O}_{2}$ at the silicon side, and the other the $\mathrm{H}_{2} \mathrm{O}_{2}$ reduction at the metal side. The second mechanism is not light responsive and is triggered by the redox decomposition of $\mathrm{H}_{2} \mathrm{O}_{2}$ exclusively at the Pt surface. We show that it is possible to enhance/suppress one mechanism over the other by tuning the surface roughness of the micromotor metal. More specifically, the actuation mechanism can be switched from light-controlled electrokinetics to light-insensitive diffusio-osmosis by only increasing the metal surface roughness. The different actuation mechanisms yield strikingly different fluid flow velocities, electric fields and light sensitivities. Consequently, these findings are very relevant and can have a remarkable impact on the design and optimization of photoactivated catalytic devices and, in general, on bimetallic or insulatingmetallic motors.
\end{abstract}

\section{Introduction}

Micro-nanomachines propelled by the self-generation of chemical gradients ${ }^{1,2}$ are drawing the attention of the research community due to their potential applications in priority areas such as nanomedicine ${ }^{3-15}$ and environmental remediation $^{12-21}$. These active objects are also very attractive due to their non-equilibrium nature, which makes them exhibit exciting phenomena such as collective motion, complex self-organization or the emergence of largescale coherent structures ${ }^{22-25}$.

Photochemically driven motors are gaining interest, as light becomes an additional tool to fine tune their motion $^{26-31}$. Although there have been many studies related to chemically driven motors with UV light ${ }^{29-40}$, very few studies are devoted to the photoactivation of motors with visible or longer wavelengths ${ }^{4-44}$. Photochemical activation with harmless and high penetration visible/near infrared light is very promising for biomedical applications.

In the last decade, many efforts have been devoted to increase motor sophistication by endowing them with multiple functionalities and tasks. However, there are still many fundamental questions related to the chemomechanical propulsion mechanisms that remain open, even more when an additional parameter such as light is incorporated. Unraveling the complex interplay of the different physical-chemical processes involved in the chemomechanical actuation is crucial to improve the levels of control and to optimize the desired applications ${ }^{45-46}$.

Evidences of the poor understanding of the propulsion mechanism can be grasped with some typical bubble-free motors in $\mathrm{H}_{2} \mathrm{O}_{2}$ solution. Common examples of these motors are the bimetallic rods or relatively small Janus microparticles composed of half metal and half insulator. The motors are propelled through phoretic processes triggered by the decomposition of $\mathrm{H}_{2} \mathrm{O}_{2}$ at their surfaces. In the case of bimetallic motors there is a big consensus that the chemomechanical actuation is rooted in an electrophoretic process $45^{-48}$. The $\mathrm{H}_{2} \mathrm{O}_{2}$ decomposition involves separate oxidation half reaction at one segment and reduction half-reaction at the other segment, with generation and consumption of protons at those segments. A local electric field is self-generated by the proton current from one segment to the other, being especially strong at the interface between both metals.

However, motors made of insulator and metal segments have opened an ongoing debate. In those cases the fuel 
decomposition takes place only at the metal side. It was initially suggested a neutral diffusiophoresis mechanism driven by the decomposition of $\mathrm{H}_{2} \mathrm{O}_{2}$ into $\mathrm{H}_{2} \mathrm{O}$ and $\mathrm{O}_{2}$ at the metal catalyst without intervention of ionic products $^{49-50}$. In that case the asymmetric distribution of reaction products would create a pressure gradient across the particle. A fluid flow would be directed towards the high pressure (high solute concentration) side inducing particle motion ${ }^{51}$. However, recent investigations revealed that the details of the propulsion mechanism are quite complex and a relevant presence of ions and electrophoretic effects (and even bulk reactions) should be taken into account to provide a more complete description of the catalytic actuation ${ }^{2-54}$.

A more complex scenario is found when motors are composed of a semiconductor and a metal catalyst. We recently reported highly efficient metal/semiconductor pumps, which could be controlled by visible light 44 . These pumps can be considered as the inverse and immobilized counterpart of swimmers. Micropumps constitute ideal platforms to acquire relevant information related to the actuation mechanisms by analyzing the fluid motion with respect to the motor surface. Our previous studies were focused on pumps made of silicon and a metal ( $\mathrm{Pt}, \mathrm{Au})$. We argued that light creates electron/hole pairs at the silicon and that electrons are transferred to the metal. As a result, light activates two separated redox reactions at the motor surface: i) $\mathrm{H}_{2} \mathrm{O}_{2}$ mediated oxidation at the semiconductor part, and ii) $\mathrm{H}_{2} \mathrm{O}_{2}$ reduction at the metal catalyst. The separated reactions give rise to a proton current from the semiconductor to the metal, thereby building up an electric field that triggers the fluid flow by electroosmosis ${ }^{44}$.

In this work, using a similar semiconductor/metal pump configuration, we show that it is possible to enhance/suppress the photoactivated and separated $\mathrm{H}_{2} \mathrm{O}_{2}$ redox reactions and convert them into light insensitive direct electrochemical decomposition of $\mathrm{H}_{2} \mathrm{O}_{2}$ on Pt. Such change in the chemical pathway can be achieved by just tuning the surface roughness of the metal. In the previous work detailed in ref. 44 , we only detected the photoactivated chemomechanical mechanism because pumps were made of very smooth platinum discs. In this paper we demonstrate that the increase of metal roughness triggers a striking change in the flow velocity and generated electric fields. That is due to a switch of the actuation mechanism from a dominant light-controlled electrokinetic process to a light-insensitive diffusio-osmotic one. These findings are very relevant, as they can have a high impact on the design and optimization of light activated catalytic motors.

\section{Results and Discussion}

Micropumps were fabricated by patterning Pt discs on p-doped silicon surfaces using electron beam lithography and platinum evaporation. The metal deposition was performed either by sputtering or electron beam evaporation to tune the surface roughness. The devices were then subjected to a mild oxygen plasma treatment to clean the surface (See Supplementary information for fabrication details). Fig. 1 illustrates a schematic picture of the micropumps under study. The figure shows atomic force microscopy images of the Pt part prepared with both deposition techniques. Devices with sputtered Pt (Fig. 1b) exhibit a roughness factor 14-fold larger than that obtained through e-beam deposition (Fig. 1a). Scanning electron microscopy (SEM) characterization also indicated a higher surface roughness on the sputtered Pt films, as can be observed in the SEM images of the Supplementary Information (Fig. $\mathrm{S}$ ). From now on, we will refer to the micropumps as r-Pt/Si for the rough sputtered ones, and s-Pt/Si for the smooth e-beam evaporated ones.

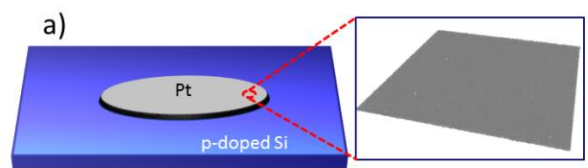

b)

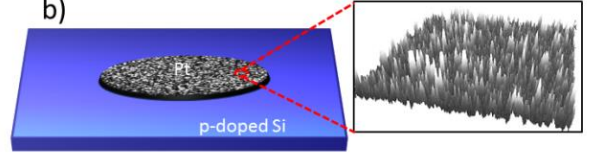

Figure 1. Schematics of the pumps with (a) smooth ebeam evaporated $\mathrm{Pt}$ and with (b) rough sputtered $\mathrm{Pt}$, together with their respective topographic AFM images taken at the same $\mathrm{z}$ scale. Sputtered Pt exhibits a roo mean square roughness $\left(R_{q}\right)$ of $5.5 \pm 0.3 \mathrm{~nm}$ whereas $R_{q}$ for electron beam deposited Pt is $0.40 \pm 0.05 \mathrm{~nm}$

The s-Pt/Si devices present the expected behavior shown in our previous study ${ }^{44}$, which pointed out a photoactivated electrochemical actuation through an electroosmotic process. According to previous results, the pumping mechanism for s-Pt/Si is illustrated in Fig. 2 a and b. The light absorbed in the Si part generates electron-hole pairs. In the vicinity of the Pt disc, the holes oxidize the $\mathrm{H}_{2} \mathrm{O}_{2}$ at the silicon/fluid interface, and the resulting electrons are transferred to the metal where the reduction of $\mathrm{H}_{2} \mathrm{O}_{2}$ takes place. During the oxidation reaction, protons are produced at the silicon region, which are consumed at the metal side. During this process a proton current is produced that generates an electric field pointing towards the Pt side, thereby dragging the fluid in the same direction. Such fluid motion pointing in the same direction as the electric field is expected for surfaces with negative zeta potentials, as the $\mathrm{Si} / \mathrm{Pt}$ case ${ }^{44}$.

The impact of Pt roughness on the pump performance is elucidated by comparing the results of fluid motion in smooth e-beam evaporated and rough sputtered $\mathrm{Pt}$ pumps illuminated by high intensity white light, and using $1 \% \mathrm{H}_{2} \mathrm{O}_{2}$ as chemical fuel. The fluid motion is followed by tracking the motion of tracer particles of opposite surface charge (zeta potentials of $\xi=-83 \mathrm{mV}$ and $\xi_{+}=46 \mathrm{mV}$ for negative and positive colloids, respectively). More details of the particle tracers can be found in the Supplementary Information. 

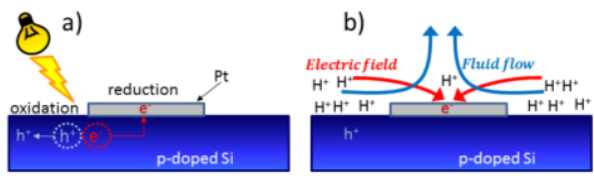

Figure 2. a) Schematic picture illustrating the photochemical activated process with oxidation and reduction taking place at the $\mathrm{Si}$ and $\mathrm{Pt}$ sides, respectively. b) Schematic representation of the proton generation and consumption as a consequence of the light activated redox processes together with the generated electric field and fluid flow direction.

Despite of their similar size and Pt thickness, the interaction of positive and negative tracers with the $\mathrm{r}-\mathrm{Pt} / \mathrm{S}$ and $\mathrm{s}-\mathrm{Pt} / \mathrm{Si}$ devices is strikingly different. In the case of $\mathrm{r}$ $\mathrm{Pt} / \mathrm{Si}$, positive tracers exhibit maximum radial velocity of $8 \mu \mathrm{m} / \mathrm{s}$. These particles move towards the Pt disc, and after crossing the Pt-Si edge, their trajectory bends upwards in the direction perpendicular to the disc, (see Fig. 3a and Movie 1). Such behavior is totally different to that previously encountered on smooth s-Pt/Si devices for similar positive tracers (Fig.3 b), which move towards the Pt disc at much higher speed (maximum velocity of -65 $\mu \mathrm{m} / \mathrm{s}$ ) and stick to it (Movie 2). Even stronger differences between rough and smooth pumps can be observed for negative tracers (Figs. $3 \mathrm{c}$ and $3 \mathrm{~d}$ ). In rough pumps, negative tracers move towards the Pt disc and they either cross the Pt edges and stick on the Pt surface or they just stick at the rims of the Pt disc (Movie 3). This behavior is surprisingly different to that found in s-Pt/Si motors, in which a repulsion band is generated at the Pt disc, thereby leaving the Pt surface and surrounding Si completely free of negative tracers (Fig. $3 \mathrm{~d}$ and Movie 4).
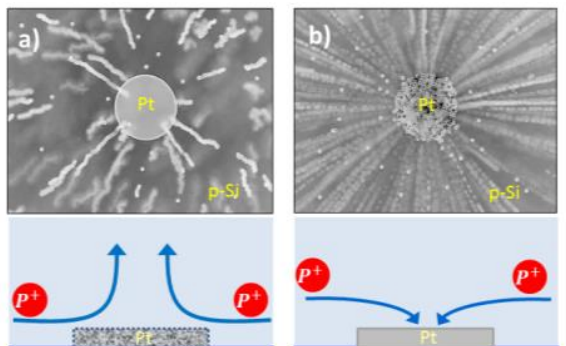

p-doped Si

p-doped Si
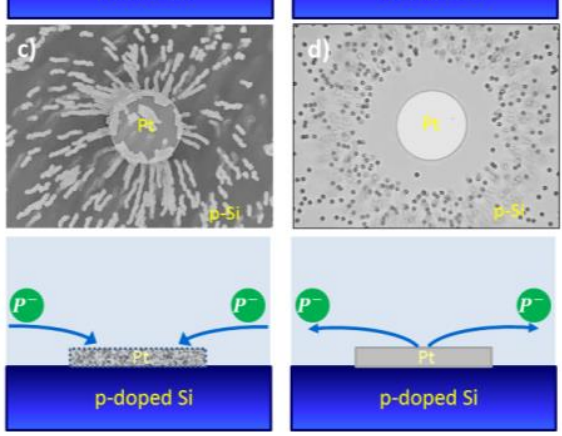

Figure 3. Interaction of positive tracers with $\mathrm{r}-\mathrm{Pt} / \mathrm{Si}$ (a) and $\mathrm{s}-\mathrm{Pt} / \mathrm{Si}$ (b) pumps. Interaction of negative tracers with $\mathrm{r}-\mathrm{Pt} / \mathrm{Si}$ (c) and s-Pt/Si (d) pumps. The schematics of the particle trajectory are also included for each pump configuration.

We also evaluated the influence of photoactivation on $\mathrm{r}-\mathrm{Pt} / \mathrm{Si}$ and $\mathrm{s}-\mathrm{Pt} / \mathrm{Si}$ devices by varying the light intensity. Figure 4 compares the tracer velocities at three different light intensities $(100 \%, 40 \%$, and below $10 \%$ with a neutral light attenuation filter). In the case of s-Pt/Si motors the radial component of the velocity of the positive tracers significantly drops as the light intensity is attenuated. In contrast, the velocity of positive tracers in the $\mathrm{r}-\mathrm{Pt} / \mathrm{Si}$ system only exhibits a very weak reduction with light intensity attenuation, and negative tracers do not show any significant variation with light intensity.

To rule out the potential influence of contaminants in the pump behavior, we have analyzed by X-ray photoelectron spectroscopy (XPS) the chemical features of the Pt films deposited by both techniques. The XPS spectra of the sputtered and electron beam evaporated Pt show similar profiles (see Supplementary Information, Fig. S2), thereby ruling out the effect of impurities in their different catalytic actuation behavior. In addition, we have analyzed the zeta potential of the sputtered Pt, giving a value of $-35 \mathrm{mV}$ (See Supplementary Information), which is within the expected zeta potential range in noble metals $(\mathrm{Au} \text { or } \mathrm{Pt})^{45,46}$. Such value also discards possible contaminants that could generate positive zeta potentials and 
induce the change of the fluid flow direction in presence of an electric field.

All these findings suggest that the actuation in the semiconductor/metal pumps is a combined effect of two different mechanisms. In the case of the s-Pt/Si pumps the catalytic actuation is dominated by electro-osmosis. The catalytic reaction generates an electric field pointing towards the Pt disc that is drastically amplified by the incident light. Such electric field triggers a fluid flow in the same direction and, in addition, induces a strong attractive force towards the Pt surface on positive tracers, and repulsive force on the negative ones (Fig. 3). In contrast, the increase of Pt roughness seems to favor the direct $\mathrm{H}_{2} \mathrm{O}_{2}$ decomposition at the entire Pt surface area, whose process is light insensitive. In that case, the liquid motion is driven by the solute concentration gradient created by such reaction at the Pt surface. This actuation behavior suggests the generation of a diffusion-osmotic process that totally changes the interaction of charged tracers with the pump.

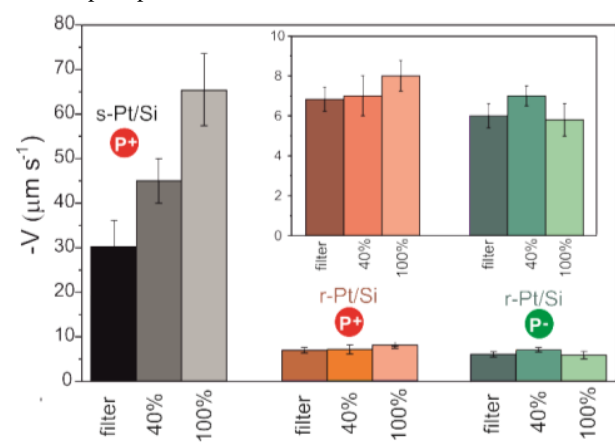

Figure 4. Influence of the photoactivation on the s-Pt/S and $\mathrm{r}-\mathrm{Pt} / \mathrm{Si}$ pumps represented by the variation of the radial component of the tracer velocity as a function of light intensity. The s-Pt/Si devices exhibit strong light responsiveness, whereas the $\mathrm{r}-\mathrm{Pt} / \mathrm{Si}$ ones are almost insensitive to light intensity variation. The figure includes a zoom of the $\mathrm{r}-\mathrm{Pt} / \mathrm{Si}$ pump as inset, for more accurate comparison.

The fluid flow and electric field values for the $\mathrm{s}-\mathrm{Pt} / \mathrm{Si}$ and $\mathrm{r}-\mathrm{Pt} / \mathrm{Si}$ pumps can be extracted by considering that the radial component of the velocity of the tracer $V_{r p}$ is the sum of two contributions: i) an electrophoretic term $\left(V_{e f}\right)$ induced by the particle electric charge, and ii) the radial fluid flow velocity $\left(V_{r f}\right)$ :

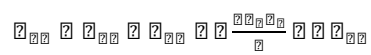

where $\varepsilon$ is the fluid permittivity, $\xi_{\mathrm{p}}$ the particle zeta potential, $E_{\mathrm{r}}$ the radial component of the electric field, and $\eta$ the fluid viscosity. Both $V_{r f}$ and $E_{r}$ can be obtained by analyzing the velocities of the tracers with opposite charge (eq. 1). The results collected in Table 1 show the pronounced decrease of both $E_{r}$ and $V_{f}$ in the rough pumps compared to the smooth ones.

Table 1 Maximum values of the radial fluid velocity $\left(V_{\text {rfmax }}\right)$ and Electric field $\left(E_{\text {rmax }}\right)$ for the different micropumps. Negative sign denotes radial inward direction of the electric field and fluid flow.

\begin{tabular}{|l|l|l|}
\hline System & $\mathrm{V}_{\text {fmax }}\left(\mu \mathrm{m} \mathrm{s}^{-1}\right)$ & $\mathrm{E}_{\mathrm{rfmax}}\left(\mathrm{Vm}^{-1}\right)$ \\
\hline $\mathrm{s}-\mathrm{Pt} / \mathrm{Si}$ & $-(43 \pm 1)$ & $-(680 \pm 1)$ \\
& & \\
\hline $\mathrm{r}-\mathrm{Pt} / \mathrm{Si}$ & $-(7 \cdot 2 \pm 1)$ & $-(24 \pm 1)$ \\
\hline $\mathrm{r}-\mathrm{Pt} / \mathrm{SiO}_{2}$ & $-(5 \cdot 4 \pm 0.8)$ & $31.7 \pm 0.8$ \\
\hline
\end{tabular}

To gain more insight into these competing mechanisms, we suppressed the photoactivated reactions, and hence their associated electric field contribution, by fabricating rough and smooth pumps on insulating $\mathrm{SiO}_{2}$. In the $s-\mathrm{Pt} / \mathrm{SiO}_{2}$ pumps, we could not capture any tangible directional motion of the positive or negative tracers in the presence of $1 \% \mathrm{H}_{2} \mathrm{O}_{2}$. However, the behavior of positive and negative tracers is dramatically different in $\mathrm{r}$ $\mathrm{Pt} / \mathrm{SiO}_{2}$ pumps (Fig. 5). Positive particles move towards the Pt disc at maximum speed of $-(4.4 \pm 0.6) \mu \mathrm{m} / \mathrm{s}$, and they drift upwards from the surface after crossing the Pt edges (Movie 5). Similar behavior is also observed with quasi-neutral particles $\left(\xi_{0}=-12 \mathrm{mV}\right)$, which approach the $\mathrm{Pt}$ disc at maximum velocities of $-(5.7 \pm 0.5) \mu \mathrm{m} / \mathrm{s}$. On the contrary, negative particles move towards the Pt disc with maximum velocities of $-(6.9 \pm 0.5) \mu \mathrm{m} / \mathrm{s}$, and then they stick to it (Movie 6). These different responses suggest the generation of an electric field in the Pt disc pointing outwards. However, the value of this electric field is too weak to enable repelling the positive tracers from the $\mathrm{Pt}$ surface. The weaker repulsive force is also due to the lower absolute value of the zeta potential in positive tracers as compared to the negative ones. As a result, the tracer motion is dominated by the fluid flow component, and is less sensitive to the generated electric fields. 

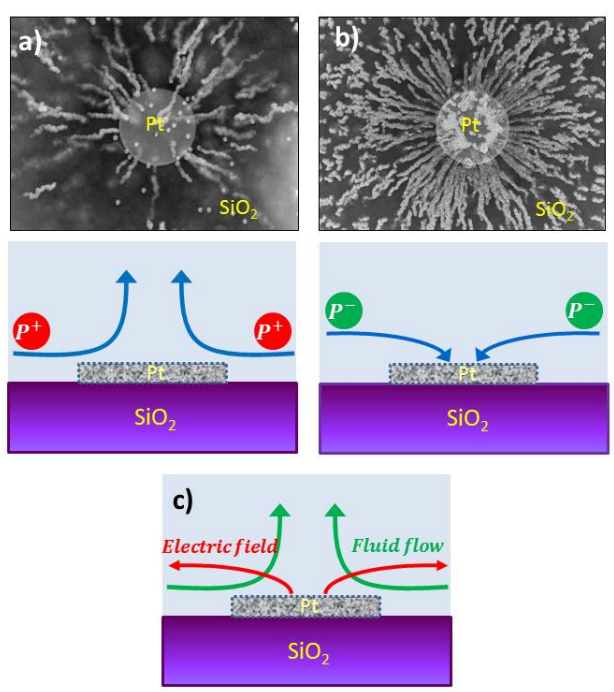

Figure 5. Interaction of positive (a) and negative (b) tracers with the $\mathrm{r}-\mathrm{Pt} / \mathrm{SiO}_{2}$ system. Schematics of the trajectories for positive and negative tracers at the rough $\mathrm{Pt} / \mathrm{SiO}_{2}$ pump are also included. c) Schematic depicting the fluid flow and electric field direction.

The quantification of $E_{r}$ and $V_{r f}$ in the rough $\mathrm{Pt} / \mathrm{SiO}_{2}$ pumps by using Eq. 1 yields $31.7 \mathrm{Vm}^{-1}$ and $-5.4 \mu \mathrm{ms}^{-1}$, respectively (Table 1 ). This analysis demonstrates the existence of an electric field in the opposite direction to that in smooth $\mathrm{Pt} / \mathrm{Si}$ pumps, i.e. pointing outwards the Pt disc. Therefore, the electric field direction is opposite to the expected direction in electro-osmotically driven pumps whose elements exhibit negative zeta potentials, as is the case of Si and Pt. In pumps with negative zeta potentials, the electric field should drive the fluid flow in the same direction according to the equation,

$$
\text { 国国国回觜回 }
$$

where $\xi_{\mathrm{s}}$ is the zeta potential of the surface $(<-4 \mathrm{omV}$ in our case due to the strong contribution of the negative surface potential of silicon) ${ }^{44}$.

The weak positive electric field in rough $\mathrm{Pt} / \mathrm{SiO}_{2}$ pumps cannot be the responsible of triggering a fluid flow in the opposite direction. Therefore, the catalytic actuation in the $\mathrm{r}-\mathrm{Pt} / \mathrm{SiO}_{2}$ pumps implies the generation of diffusioosmosis processes induced by the direct decomposition of $\mathrm{H}_{2} \mathrm{O}_{2}$ at the rough Pt surface. As a result, the fluid moves towards the region where the reaction takes place, i.e. the Pt disc where the solute concentration and the pressure are higher.

The existence of an electric field in the metal/insulator pumps is in line with previous findings in metal/insulator Janus particles, which pointed out the generation of an electric field induced by charged reactions and ionic ef-

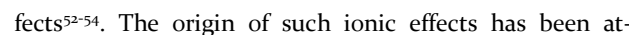
tributed to different sources. It was proposed that asymmetries in the metal catalyst, such as different metal thicknesses, could yield to reactions involving charged intermediates ${ }^{33}$. That could be a plausible explanation for rod and sphere like structures capped by the catalyst, in which a thickness gradient with thicker catalyst layer at the pole than at the equator sides is expected after metal evaporation. However, such thickness asymmetries are expected to be negligible in the flat configuration of our pumps. Other studies suggested the potential dissociation of $\mathrm{H}_{2} \mathrm{O}_{2}$ and water in ions at the metal surface and in the bulk solution as additional sources for a self-generated electric field54. However, the generated electric field in the $\mathrm{r}-\mathrm{Pt} / \mathrm{SiO}_{2}$ pumps is not the driving force of the fluid flow, as it should generate the electrokinetic fluid flow in the opposite direction to what it is observed. Therefore, the electrokinetic propulsion mechanism can be discarded in the metal/insulator pump. The chemical origin of the electric field is not well known at this moment but future studies including photoemission experiments are pursued to better understand the $\mathrm{Si} / \mathrm{Pt}$ interface and the precise chemical reaction schemes that could yield to an electric field in such conditions.

\section{Conclusion}

The findings in insulating $\mathrm{SiO}_{2} / \mathrm{Pt}$ pumps demonstrate that at least two chemo-mechanical mechanisms are behind the liquid propulsion in $\mathrm{Si} / \mathrm{Pt}$ pumps. The first one is an electro-osmotic process due to a photoactivated reaction taken place in two separated redox reactions: the oxidation of $\mathrm{H}_{2} \mathrm{O}_{2}$ at the $\mathrm{Si}$ side and the reduction of $\mathrm{H}_{2} \mathrm{O}_{2}$ at the $\mathrm{Pt}$ side. The induced proton current from $\mathrm{Si}$ towards Pt in these reactions generates the electric field towards Pt that drags the fluid towards the metal structure. In contrast, the second propulsion mechanism features diffusio-osmotic characteristics, with $\mathrm{H}_{2} \mathrm{O}_{2}$ decomposition taking place mainly at the $\mathrm{Pt}$ region. This decomposition process triggers the fluid flow towards the $\mathrm{Pt}$ structure to compensate the higher solute concentration and higher osmotic pressure. This latter mechanism is not light responsive. The dominance of one chemomechanical mechanism over the other depends on the catalyst roughness. In smooth $\mathrm{Pt} / \mathrm{Si}$ pumps the photoactivated electro-osmotic mechanism prevails, whereas the diffusioosmotic and light insensitive one dominates in rough $\mathrm{Si} / \mathrm{Pt}$. As a consequence, to develop highly efficient light sensitive engines, the generation of separated redox interfacial reactions between $\mathrm{Si}$ and $\mathrm{Pt}$ should be maximized by minimizing metal roughness and increasing the $\mathrm{Si} / \mathrm{Pt}$ perimeter. These results are then crucial for the design of new photoactivated nanoswimmers/pumps controlled by visible and near infrared light. More specifically, these findings can have direct impact on light controlled motion of semiconductor/metal micro/nanostructures, in which the metal can act as a single catalyst by itself, or as part of a coupled catalytic process with the semiconductor 


\section{ASSOCIATED CONTENT}

Supporting Information

Details on Pump fabrication, particle tracers and optical tracking, platinum film characterization by SEM, XPS and zeta potential.

The following AVI Movies are attached:

Movie 1: Motion of positive tracers on $\mathrm{r}-\mathrm{Pt} / \mathrm{Si}$ under white light exposure.

Movie 2: Motion of positive tracers on s-Pt/Si under white light exposure

Movie 3: Motion of negative tracers on $\mathrm{r}-\mathrm{Pt} / \mathrm{Si}$ under white light exposure..

Movie 4: Motion of negative tracers on s-Pt/Si under white light exposure.

Movie 5: Motion of positive tracers on $\mathrm{r}-\mathrm{Pt} / \mathrm{SiO}_{2}$

Movie 6: Motion of negative tracers on $\mathrm{r}-\mathrm{Pt} / \mathrm{SiO}_{2}$

\section{AUTHOR INFORMATION}

\section{Corresponding Author}

* M.J. Esplandiu,mariajose.esplandiu@icnz.cat; B. Sepulveda, borja.sepulveda@icn2.cat.

\section{Author Contributions}

The manuscript was written through contributions of all authors. / All authors have given approval to the final version of the manuscript.

\section{Funding sources}

This research was supported by the Spanish Ministry of Economy and Competitiveness, MINECO (under contracts No. MAT2015-68307-P and M-ERA.NET project PCIN-2016093). The ICN2 is funded by the CERCA programme / Generalitat de Catalunya. The ICN2 is supported by the Severo Ochoa programme of the Spanish Ministry of Economy, Industry and Competitiveness (MINECO, grant no. SEV-2013 0295).)

\section{ACKNOWLEDGMENT}

This research was partially supported by the Spanish Ministry of Economy and Competitiveness, MINECO (under Contracts No. MAT2015-68307-P and M-ERA.NET project PCIN2016-093). The ICN2 is funded by the CERCA programme Generalitat de Catalunya. The ICN2 is supported by the Severo Ochoa programme of the Spanish Ministry of Economy, Industry and Competitiveness (MINECO, grant no. SEV2013-0295). K.Z. acknowledges support from Severo Ochoa fellowship.

\section{REFERENCES}

(1) Ozin, G.A.; Manners, I.; Fournier-Bidoz, S.; Arsenault, A Dream Nanomachines. Adv. Mater. 2005, 17, 3011-3018.

2) Paxton, W.F.; Kistler, K.C.; Olmeda, C.C.; Sen, A.; St. Angelo S.K.; Cao, Y.; Mallouk, T.E.; Lammert, P.E.; Crespi, V.H. Catalytic Nanomotors: Autonomous Movement of Striped Nanorods. J. Am. Chem. Soc., 2004, 126, 13424-13431.
(3) Li, J.; Esteban- Fernandez de Avila, B.; Gao, W.; Zhang, L.; Wang, J. Micro/Nanorobots for biomedicine: Delivery, surgery, sensing and detoxification. Science Robotics 2o17, 2, aam6431.

(4) Hong, W.; Pumera, M. Emerging materials for the fabrication of micro/nanomotors. Nanoscale 2017, 9, 2109-2116.

(5) Esteban-Fernandez de Avila, B.; Angsantikul, P.; Li, J.; Lopez-Ramirez, M.A.; Ramirez-Herrera, D.E., Thamphiwatana, S.; Chen, C.; Delezuk, J.; Samakapiruk, R.; Ramez, V.; Zhang, L.; Wang, J. Micromotor enabled active drug delivery for in vivo treatment of stomach infection. Nat. Comm. 2017. DOI 10.1038/s41467-017-00309-w.

(6) Medina-Sanchez, M.; Schwarz, L.; Hebenstreit, F Schmidt, O.G. Cellular cargo delivery: toward assisted fertilization by sperm carrying micromotors. Nano Lett. 2016, 16, 555-561.

(7) Magadanz, V.; Guix, M.; Hebenstreit, F.; Schmidt, O.G. Dynamic polymeric microtubes for the remote-controlled capture, guidance and release of sperm cells. Adv. Mater. 2016, 28, 4084-4089.

(8) Singh, W.; Kaufmann, K.; Esteban-Fernandez de Avila, B.; Karshalev, E.; Wang, J. Molybdenum disulfide-based tubula microengines: towards biomedical applications. Adv. Funct Mater. 2016, 26, 6270-6278.

(9) Gao, W.; Wang, J.; Synthethic micro/nanomotors in drug delivery. Nanoscale 2014, 6, 10486-10494.

(10) Solovev, A.A.; Sanchez, S.; Mei, Y.; Schmidt, O.G. Tunable catalytic tubular micro-pumps operating at low concentrations of hydrogen peroxide. PCCP 2011, 13, 10131-10135.

(11) Magdanz, V.; Sanchez, S.; Schmidt, O.G. Development of a Sperm-Flagella Driven Micro-Bio-Robot. Adv. Mater. 2013, 25, $6581-6588$.

(12) Wang, H: Pumera, M. Fabrication of Micro/Nanoscale Motors. Chem. Rev. 2015, 115, 8704-8735.

(13) Moo, J.G.S.; Mayorga-Martinez, C.C.; Wang H.; Khezri, B.; Teo, W.Z.; Pumera, M. Nano/Microrobots Meet Electrochemistry. Adv. Funct. Mater. 2017, 27, 1604759.

(14) Xu, T; Xu, L.; Zhang X. Ultrasound propulsion of micronanomotors. Appl. Mater. Today 2017, 9, 493-503.

(15) Jurado-Sánchez, B.; Pacheco, M.; Hormigos, M.; Escarpa A. Perspectives on Janus micromotors: Materials and applications. Appl. Mater. Today 2017, 9, 407-418.

(16) Vilela, D.; Stanton, M.M.; Parmar, J.; Sanchez, S. Microbots decorated with silver nanoparticles kill bacteria in aqueous media. ACS Appl. Mater. Interfaces. 2017, 9, 22093-22100.

(17) Soler, L.; Sanchez, S. Catalytic Nanomotors for Environmental Monitoring and Water Remediation. Nanoscale 2014, 6, 7175 7182

(18) Vilela, D.; Parmar, J.; Zeng, Y.F.; Zhao, Y.L.; Sanchez, S. Graphene based microbots for toxic heavy metal removal and recovery from water. Nano Lett. 2016, 4, 2860-2866.

(19) Wang, H. Khezri, B; Pumera, M. DNA-functionalized self-propelled micromachines for environmental remediation. CHEM. 2016, 1, 473-481.

(20) Gao, W.; Wang, J. The Environmental Impact of Micro/Nanomachines. A Review. ACS Nano 2014, 8, 3170-318o.

(21) Li, J.; Singh, V.V.; Sattayasamitsathit, S.; Orozco, J.; Kaufmann, K.; Dong, R.; Gao, W.; Jurado-Sanchez, B.; Fedorak, Y. Wang, J. Water-Driven Micromotors for Rapid Photocatalytic Degradation of Biological and Chemical Warfare Agents. ACS Nano 2014, 8, 11118-11125.

(22) Illien, P.; Golestanian, R.; Sen, A. Fueled motion: phoretic motility and collective behavior of active colloids. Chem. Soc Rev. 2017, 18, 5508-5518.

(23) Vutukuri, H. Rao; Preisler, Z.; Besseling, T.H., van Blaaderen, A.; Dijkstra, M.; Huck, W.T.D., Dynamic self-organization of side propelling colloidal rods: experiments and simulations. Soft Matter 2016, 12, 9657-9665. 
(24) Alarcon, F.; Valeriani, C.; Pagonabarraga, I. Morphology of clusters of attractive dry and wet self-propelled spherical particle suspensions. Soft Matter 2017, 13, 814-826.

(25) Wang, W.; Duan, W.T.; Ahmed, S.; Sen, A.; Mallouk, T.E. From one to many: dynamic and collective behavior of selfpropelled colloidal motors. Acc. Chem. Res. 2015, 48, 1938-1946.

(26) Safdar, M.; Simmchen, J.; Janis, J. Light-driven micro and nanomotors for environmental remediation. Env. Sci. Nano 2017 , 4, 1602-1616.

(27) Jang, B.; Hong, A.; Kang, H.E.; Alcantara, C.; Charreyron, S. Mushtaq, F. Pellicer, E. Buchel, R. Sort, J.; Lee, S.S.; Nelson, B.J.; Pane, S. Multiwavelength Light-Responsive $\mathrm{Au} / \mathrm{B}_{-\mathrm{TiO}}$ Janus Micromotors. ACS Nano 2017, 11, 6146-6145.

(28) Jurado-Sanchez, B.; Wang, J.; Escarpa, A. Ultrafast Nanocrystals Decorated Micromotors for On-Site Dynamic Chemical Processes. ACS App. Mater. Interfaces 2016, 8, 19618-19625.

(29) Dai, B.; Wang, J.; Xiong, Z.; Zhan, X.; Dai, W.; Li, C.; Feng, S.; Tang, J. Programmable artificial phototactic microswimmer Nat. Nanotech. 2016, 11, 1087-1092.

(30) Tang, S.; Sivan, V.; Khoshmanesh, K.; O'Mullane, A.P. Tang, X; Gol, B.; Eshtiaghi, N.; Lieder, F.; Petersen, F.; Mitchell, A.; Kalantar-zadeh, K. Electrochemically induced actuation of liquid metal marbles. Nanoscale 2013, 5, 5949-5957.

(31) Tang, X.; Tang, S.; Sivan, V.; Zhang, W.; Mitchell, A.; Kalantar-zadeh, K.; Khoshmanesh, K. Photochemically induced motion of liquid metal marbles. Appl. Phys. Lett. 2013, 103 174104 .

(32) Simmchen, J.; Baeza, A.; Miguel-López, A.; Stanton, M.M. Vallet-Regi, M.; Ruiz-Molina, D.; Sánchez, S. Dynamics of novel photoactive $\mathrm{AgCl}$ microstars and their environmental applications. ChemNanoMat. 2017, 3, 65-71.

33) Wang, S.; Jiang, Z; Ouyang, S.; Dai, Z.; Wang, T. Internally/Externally Bubble-Propelled Photocatalytic Tubular Nanomotors for Efficient Water Cleaning. ACS Appl. Mater. Interfaces 2017, 28, 23974-23982.

34) Zhang, Q.; Dong, R.; Wu, Y.; Gao, W.; He, Z.; Ren, B. Light-Driven Au-WO3@C Janus Micromotors for Rapid Photodegradation of Dye Pollutants. ACS Appl. Mater. Interfaces 2017 $4674-4683$.

35) Akhavan, O.; Saadati, M.; Jannesari, M. Graphene Jet Nanomotors in Remote Controllable Self-Propulsion Swimmers in Pure Water. Nano Lett. 2o16, 16, 5619-5630.

(36) Dong, R.; Zhang, Q; Gao, W.; Pei, A.; Ren, B. Highly Efficient Light-Driven TiO2-Au Janus Micromotors. ACS Nano 2016, $10,839-844$.

(37) Ibele, M.; Mallouk, T.E.; Sen, A. Schooling Behavior of Light-Powered Autonomous Micromotors in Water. Angew. Chem. Int. Ed. 2009, 48, 3308-3312.

(38) Hong Y ; Diaz, M. Córdova-Figueroa, U.M. Sen, A Light-Driven Titanium Dioxide Based Reversible Microfireworks and Micromotor/Micropump Systems. Adv. Funct. Mater. 2010 20, 1-9.

(39) Yadav, V.; Zhang H.; Pavlick, R.; Sen A. Triggered "On/Off" Micropumps and Colloidal Photodiode. J. Am. Chem. Soc. 2012, 134, 15688-15691.

(40) Giudicatti, S.; Marz, S.M.; Soler, L.; Madani, A.; Jorgensen, M.R.; Sanchez, S.; Schmidt, O.G. Photoactive Rolled-up $\mathrm{TiO}_{2}$ Microtubes: Fabrication, Characterization and Applications. J. Mater. Chem. C, 2014, 2, 5892-5901.

(41) Zhou, D.; Li, Y.C.; Xu, P.; Ren, L.; Zhang, G.; Mallouk, T.E.; $\mathrm{Li}$, L. Visible-light driven Si-Au micromotors in water and organic solvents. Nanoscale 2017, 32, 11434-11438.

(42) Zhou, D.; Li, Y.C.; Xu, P.; Ren, L.; McCool, N.S.; Li, L.; Wang, W.; Mallouk, T.E. Visible-light controlled catalytic $\mathrm{Cu}_{2} \mathrm{O}-$ Au micromotors. Nanoscale 2017, 9, 75-78.
(43) Dong, E.; Hu, Y.; Wu, Y.; Gao, W.; Ren, B.; Wang, Q; Cai, Y. Visible-Light-Driven BiOI-Based Janus Micromotor in Pure Water. J. Am. Chem. Soc. 2017, 139, 1722-1725.

(44) Esplandiu, M.J.; Afshar Farniya, A.; Bachtold, A. Siliconbased chemical motors: an efficient pump for triggering and guiding fluid motion using visible light. ACS Nano 2015, 9, 11234-

(45) Afshar-Farniya, A.; Esplandiu, M.J.; Reguera, D.; Bachtold A. Imaging proton concentration and mapping the spatial distribution of the electric field of catalytic micropumps. Phys. Rev. Lett. 2013, 111, 168301.

(46) Esplandiu, M.J.; Afshar Farniya, A.; Reguera, D. Key parameters controlling the performance of catalytic motors. J. Chem. Phys. 2016, 144, 124702.

(47) Paxton, W.F.; Baker, Kline, T.R.; Wang, Y.; Mallouk, T.E Sen, A. Catalytically induced electrokinetics for motors and pumps. J. Am. Chem. Soc. 2006, 128, 14881-14888.

(48) Moran, J.L.; Posner, J.D. Electrokinetic locomotion due to reaction-induced charge auto-electrophoresis. J. Fluid Mech 2011, 680, 31-66.

(49) Golestanian, R.; Liverpool, T.B.; Adjari, A. Propulsion of molecular machine by asymmetric distribution of reaction products. Phys. Rev. Lett. 2005, 94, 220801.

(50) Howse, J.R.; Jones, R.A.L.; Ryan, A.J.; Gough T.; Vafabakhsh, R.; Golestanian, R. Self-Motile Colloidal Particles from Directed Propulsion to Random Walk, Phys. Rev. Lett. 2007, 99 048102 .

(51) Anderson, J.L. Colloid Transport by Interfacial Forces, Annu. Rev. Fluid Mech. 1989, 21, 61-99.

(52) Brown, A.; Poon, W. Ionic Effects in Self-Propelled PtCoated Janus Swimmers, Soft Matter. 2014, 10, 4016-4027.

(53) Ebbens, S.; Gregory, D.A.; Dunderdale, G.; Howse, J.R.; Ibrahim Y.; Liverpool, T.B.; Golestanian, R. Electrokinetic Effects in Catalytic Pt-Insulator Janus Swimmers, Europhys. Lett. 2014 106,58003 .

(54) Brown, A.T.; Poon, W.C.K.; Holm, C.; de Graaf, J. Ionic screening and dissociation are crucial for understanding chemical self-propulsion in polar solvents. Soft Matter 2017, 13, 12001222. 
SYNOPSIS TOC

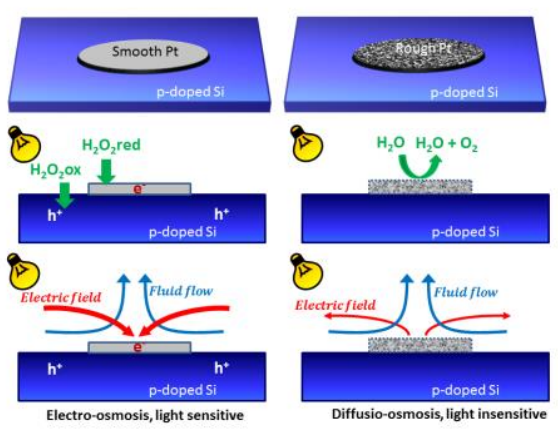




\section{Supplementary Information}

\section{Photochemically activated motors: from electrokinetic to diffusion motion control}

Kuan Zhang, Jordi Fraxedas, Borja Sepulveda, Maria J. Esplandiu

\section{Pump fabrication, particle tracers and optical tracking}

Micropumps were fabricated by patterning Pt discs with a diameter of $50 \mu \mathrm{m}$ and a thickness of 50 $\mathrm{nm}$ on p-doped silicon surfaces $(10-20 \mathrm{ohm} \mathrm{cm})$ by electron beam lithography, followed by platinum deposition either by sputtering or electron beam evaporation. In the case of the e-beam evaporation a very thin adhesion layer of chromium was deposited between the metal disc and the silicon. The devices were subjected to 1 min of plasma cleaning $(360 \mathrm{~W})$ to remove organic contamination. An 8 $\mathrm{mm}$ diameter and $0.12 \mathrm{~mm}$ thick gasket-like spacer (Invitrogen) was placed on top of the wafer patterned with pumps. A $1 \%$ hydrogen peroxide solution containing differently charged microparticles was added to the vacant space created by the gasket. The wafer was immediately capped with a thin glass cover. We used three different tracing particles: $2 \mu \mathrm{m}$ diameter polystyrene spheres (Kisker Biotech $\mathrm{GmbH} \& \mathrm{Co}$ ) as quasi- neutral particles, $2 \mu \mathrm{m}$ diameter polystyrene spheres functionalized with amidine groups (Invitrogen) as positive tracers, and $2 \mu \mathrm{m}$ diameter silica spheres (SigmaAldrich) as negative tracers. The zeta potentials were determined in a $1 \% \mathrm{H} 2 \mathrm{O} 2$ solution with a Malvern ZetaSizer Nano ZS Instrument. The particle motion was optically recorded with a rate of 5 frames per second and analyzed with the Diatrack software to determine the radial velocity of tracers.

\section{Characterization of Pt films}

\section{SEM Images}
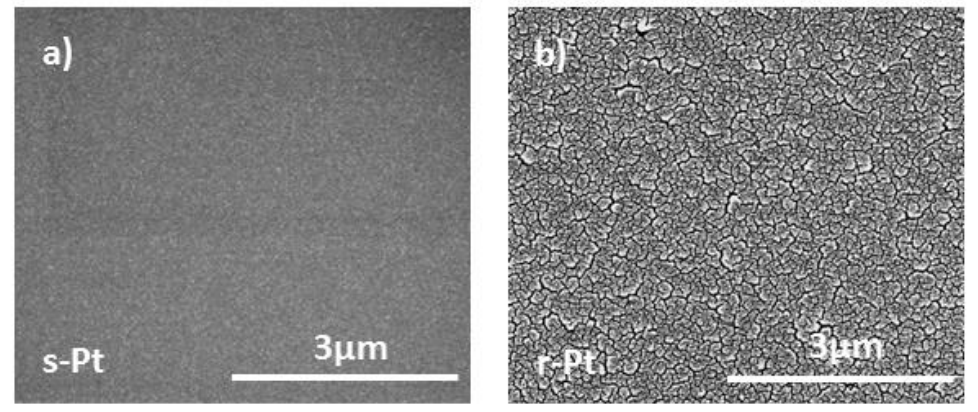

Figure S1. SEM images corresponding to the smooth Pt films evaporated by e-beam deposition (a) and to the rough Pt films deposited by sputtering (b). Both images were taken at the same magnification.

\section{XPS spectra}




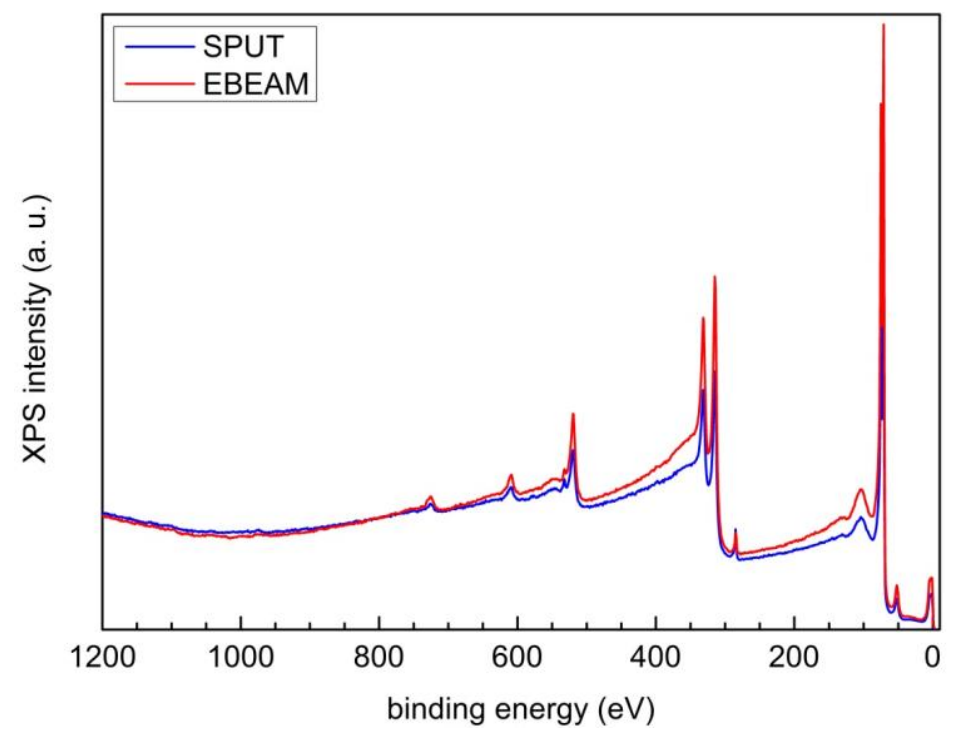

Figure S2. Survey XPS spectra of platinum films deposited on silicon substrates by sputtering (continuous blue line) and e-beam evaporation (continuous red line), respectively. The samples were measured as-received and without previous exposure to oxygen plasma. The experiments were performed with a PHOIBOS150 hemispherical analyser hosted in a UHV system with a base pressure of 5x10-10 mbar and using monochromatic AlKa radiation $(1486.6 \mathrm{eV})$. The spectra reveal carbon contamination $(\mathrm{C} 1 \mathrm{~s}$ line at $285 \mathrm{eV}$ ) arising from the unavoidable exposure to the atmosphere and a weak nitrogen signal at about $400 \mathrm{eV}$, again due to exposure to air during transport from the deposition equipment to the XPS system. Both samples were exposed less than 30 minutes to the atmosphere. No other contaminants can be detected in spite of the high surface sensitivity of the XPS technique.

\section{Zeta potential of Pt deposited by sputtering}

A rough estimation of the zeta potential of sputtered Pt was obtained with dynamic light scattering (Malvern ZetaSizer Nano ZS Instrument) using an aqueous dispersion of silicon rods coated with sputtered Pt. 